\title{
The effect of conjugated linoleic acid on oxidative stress and matrix metalloproteinases 2 and 9 in patients with COPD
}

This article was published in the following Dove Press journal: International Journal of COPD

\author{
Somaieh Matin' \\ Ali Nemati ${ }^{2}$ \\ Hassan Ghobadi ${ }^{3}$ \\ Reza Alipanah-Moghadam ${ }^{4}$ \\ Lotfollah Rezagholizadeh ${ }^{4}$ \\ 'Department of Internal Medicine, \\ Emam Khomeini Hospital, Ardabil \\ University of Medical Sciences, \\ Ardabil, Iran; ${ }^{2}$ Biochemistry and \\ Nutrition Department, Ardabil \\ University of Medical Sciences, \\ Ardabil, Iran; ${ }^{3}$ Department of Internal \\ Medicine (Pulmonary Division), Emam \\ Khomeini Hospital, Ardabil University \\ of Medical Sciences, Ardabil, Iran; \\ ${ }^{4}$ Department of Biochemistry, School \\ of Medicine, Ardabil University of \\ Medical Sciences, Ardabil, Iran
}

Background: Natural antioxidants in foods may be used in prevention and treatment of oxidative stress and inflammation in COPD. Therefore, this study aimed to evaluate the effect of conjugated linoleic acid (CLA) supplement as natural antioxidants on oxidative stress levels, and MMP2 and MMP9 serum levels in COPD patients.

Materials and methods: This clinical trial study was conducted on 90 (supplement group $=45$ and control group=45) COPD patients in Ardabil city, Iran, in 2015. After obtaining written consent, general information was collected from each patient using a validated and reliable questionnaire. Supplement group received $3.2 \mathrm{~g}$ of CLA and those in the control group were given $3.2 \mathrm{~g}$ of placebo for 6 weeks on a daily basis. Fasting blood samples were taken from all of the patients for testing of malondialdehyde (MDA), MMP2, and MMP9 levels at the beginning and end of the study. Data were analyzed using Kolmogorov-Smirnov test, independent samples $t$-test, paired sample $t$-test, chi-square test, and ANOVA.

Results: There were no significant differences between the two groups with regard to mean age, smoking status, and serum level of MDA at the beginning of the study. In the supplement group, the serum level of MDA decreased significantly at the end of the 6th week compared to that in the beginning of the study ( $p=0.0004)$, while in the placebo group, the difference was found to be insignificant. The serum level of MMP9 decreased significantly in the supplement group, while in the placebo group its level increased significantly as compared to that at the beginning of the study $(p<0.05)$. The serum levels of MMP2 indicated no significant differences between the two groups neither at the beginning nor at the end of the study.

Conclusion: These findings indicated that CLA supplementation may be helpful for COPD patients through inhibiting the production of oxidative stress and controlling MMP9 serum levels.

Keywords: COPD, CLA, oxidative stress, MMP2, MMP9

\section{Introduction}

COPD is the most prevalent chronic respiratory disease and one of the main causes of death throughout the world. It is characterized by progressive and irreversible airflow obstruction. ${ }^{1}$ From among the mechanisms involved in the pathogenesis of this disease, oxidative stress and inflammation have attracted increasing attention. ${ }^{2}$ Oxidative stress is indicative of an increase in the production of various types of free radicals of oxygen from inflammatory cells and the inability of the biological system to detoxify them or repair their damaging effects, thus resulting in oxidative damage
Correspondence: Ali Nemat

Biochemistry and Nutrition Department, Ardabil University of Medical Sciences,

Daneshgah Street, Ardabil, Iran

Tel +9845335I 0052

Fax +9845335I 3776

Email ali.nemati@arums.ac.ir 
to cells, tissues, and organs of the body. ${ }^{3}$ The increase of oxidative stress in lungs causes an increase in the transcription of inflammatory genes, protease activity, mucus secretion, and resistance to glucocorticoid. ${ }^{4}$ An increase in the level of free radicals of oxygen leads to an augment, either directly or through lipid peroxidation products, in the inflammatory response, which has now been recognized as the main pathogenic factor in the progression of COPD and the induction of matrix metalloproteinase (MMP) expressions. ${ }^{5}$ MMPs are a large family of protease enzymes, and 26 types have been recognized to date. It seems that MMPs have the most important role in the destruction and synthesis of collagenrich tissues in different physiological and pathological conditions. ${ }^{6}$ Among the enzymes in MMP family, the two enzymes of MMP2 (72 kDa gelatinase) and MMP9 (92 kDa gelatinase) have highest activity. MMP9 exists in low levels in the lung tissues of healthy humans. But, its level increases in severe pulmonary diseases such as asthma, primary pulmonary fibrosis, COPD, and cystic fibrosis leading to an increase in disease severity and resistance to treatment. ${ }^{7-9}$ In a study, an increase in the level of MMP2 was observed in the sputum samples taken from patients with asthma and COPD, which was statistically significant compared to the patients in control group. ${ }^{10}$ The destruction of the elasticity of the alveolar wall in COPD and its false reconstruction due to increased proteolytic activity result in the loss of alveolar function and disease progression. For this reason, MMPs have nowadays attracted increasing attention since they can have a potential role in the destruction and reconstruction of damaged alveoli. ${ }^{11}$ Regarding the role of oxidative stress in the pathogenesis of COPD, the use of antioxidant drugs as a new strategy for the treatment and prevention of this disease has become important. ${ }^{12}$ One of the supplements with antioxidant properties that has recently been focused on by many researchers due to its beneficial effects on human health condition is conjugated linoleic acid (CLA), which can be used together with usual treatments. ${ }^{12}$ CLA is a fatty acid with multiple double bonds which naturally exists in animal resources. ${ }^{13}$ The CLA supplements with different isomers can be used as antioxidant, anti-inflammatory, anticancer, and anti-atherosclerosis substances; they can also be used as a stimulant of immune system and growth factor, for changing of body composition, and so on. ${ }^{14,15}$ Although a number of studies have demonstrated possible benefits of CLA intake on inflammatory factors, ${ }^{16,17}$ there is not enough evidence to establish positive effects of CLA consumption in reducing oxidative stress and inflammatory factors in the COPD subjects. There are no studies addressing the effect of CLA supplement on the levels of oxidative stress indices and serum levels of MMPs in COPD patients. Therefore, this study aimed to evaluate the effect of CLA supplement on the levels of oxidative stress, MMP2, and MMP9 in COPD patients.

\section{Materials and methods}

This study was a double-blind clinical trial which was conducted on COPD patients in the Pulmonary Department at Imam Khomeini Hospital, Ardabil city, Iran, in 2015. At the beginning of the study, 168 male patients within the age range of 40-80 years were selected based on the criteria proposed by American Thoracic Society for having COPD, that is, coughing, phlegm production, and chronic dyspnea together with spirometry results with forced expiratory volume in first second $\left(\mathrm{FEV}_{1}\right) /$ forced vital capacity $(\mathrm{FVC})$ ratio below $70 \%$. Seventy-five patients, who were suffering from other chronic diseases such as cardiovascular diseases, diabetes, malignancies, etc, were excluded. Three patients were excluded during the study due to lack of cooperation and abuse of CLA supplements. Written informed consent form was obtained from each patient before the study. In order to collect the needed information about the patients such as their name, surname, age, education level, job, place of residence, smoking history and abandonment, and the number of exacerbations of disease during the previous year, a validated and reliable questionnaire was used for each patient. Finally, 90 patients (supplement group $=45$ and control group $=45$ ) were evaluated. This research was ethically approved in the research department of Ardabil University of Medical Sciences (Iran) with the code of "Arums.REC.93.56" and recorded in the Clinical Trial Center of Iran with the code of IRCT2015080823559N1.

\section{Supplementation}

The patients in the supplement group received $3.2 \mathrm{~g}$ of CLA in softgel capsules (with $80 \%$ purity), which include c9-t11 and c12-t10 isomers (50/50) and are manufactured in the form of tablets in the US with Nutrex research company the commercial name of Nutrex (Nutrex Reaserch Inc., Oviedo, FL, USA), while the control group received the same amount of placebo for 6 weeks. Before the beginning of the study, the containers of the CLA supplement and placebo were coded with letters A and B, respectively, to make sure that neither the interviewers nor the patients knew which group received which type of supplement, and therefore the process was double-blind.

\section{Biochemical information}

At the beginning of the study and at the end of the 6th week, $3 \mathrm{~mL}$ of venous blood samples were taken from all of the 
patients after 12 hours of fasting and gently transferred to test tubes. Then, the samples were labeled and transferred to the laboratory. After that, they were centrifuged for 10 minutes at 4,000 rpm to separate the serum. Finally, the samples were distributed in four micro-tubes and kept at the temperature of $-80^{\circ} \mathrm{C}$ for malondialdehyde (MDA), MMP2, and MMP9 tests.

The serum level of MDA was assessed by thiobarbituric acid reactive substances test using the reaction of thiobarbituric acid. MDA is one of the oxidative stress biomarkers, ${ }^{18}$ and it has been shown that there is an association between serum levels and COPD severity. ${ }^{19,20}$ The serum samples of patients were first heated with thiobarbituric acid under acidic conditions according to the instructions provided by the kit manufacturer and then cooled down. Afterward, the resulting color combination in n-butanol was extracted. The absorbance of the pink color was measured at $530 \mathrm{~nm}$. Serum MMP2 and MMP9 levels were assayed by standard enzyme-linked immunosorbent assay (ELISA) kits (Boster, Wuhan, China). Serum samples were diluted 1:100 according to the manufacturer's specifications for MMP9 and MMP2 assay. For the accuracy of assessment, the experiments were performed in duplicate. The colors formed were read at the wavelengths of 450 and $630 \mathrm{~nm}$ by Anhos 2000 microplate reader, and the sample concentrations were calculated using Multcalc Software (Wallac, Turku, Finland).

\section{Patients' follow-up}

In order to both control the consumption of supplementation and prevent the loss of samples, the follow-up was also done through face-to-face visits, in addition to making telephone calls. The pills needed for 6 weeks were given to the patients. They were asked to return the remaining pills if they stopped consuming them. In the following visits, $97 \%$ of the patients reported to have taken their pills regularly and were studied to find out the effects of the supplement on their disease. The compliance of patients in terms of taking their supplements was evaluated based on the number of remaining pills at the end of the 6th week of the study. The researcher provided the patients with some information about the possible complications of the supplement and gave them his phone number to contact him if they encountered any problem.

\section{Data analysis and statistical methods}

In order to check the normality of the data related to each variable, Kolmogorov-Smirnov test was employed. The information in the checklists was then transferred to SPSS Software (version 16), and in order to find the relationship between different variables, independent samples $t$-test, paired sample $t$-test, chi-square test, and ANOVA were performed. The significance level was taken to be 0.05 for all the tests. The results were reported in the form of mean \pm standard deviation.

\section{Results}

Demographic information and risk factors related to the patients and their comparison between the two groups are presented in Tables 1 and 2. Most of the patients in this study were urban residents. Most of the patients were farmers, and most of the subjects were illiterate. Based on the chi-square test, there was no significant difference with regard to variables (such as $\mathrm{FEV}_{1}$ percentage, age,

Table I Demographic information of the COPD patients $(n=90)$

\begin{tabular}{|c|c|c|c|c|c|}
\hline \multirow[t]{2}{*}{ Variables } & \multicolumn{2}{|c|}{ Supplement group $(n=45)$} & \multicolumn{2}{|c|}{ Placebo group $(n=45)$} & \multirow[t]{2}{*}{ p-value } \\
\hline & Number & Percentage & Number & Percentage & \\
\hline Job & & & & & $p>0.05$ (NS) \\
\hline Employee & 10 & 22.22 & II & 24.44 & \\
\hline Farmer & 20 & 44.22 & 15 & 33.33 & \\
\hline Worker & 5 & II.II & 4 & 8.88 & \\
\hline Baker & 2 & 4.44 & 2 & 4.44 & \\
\hline Carpet weaver & 1 & 2.22 & 1 & 2.22 & \\
\hline Other & 7 & 15.55 & 12 & 26.66 & \\
\hline Education level & & & & & $p>0.05$ (NS) \\
\hline Illiterate & 20 & 44.44 & 18 & 40 & \\
\hline Less than a high school diploma & 17 & 37.7 & 18 & 40 & \\
\hline Diploma of high school & 8 & 17.8 & 8 & 17.8 & \\
\hline Academic & 0 & 0 & I & 2.2 & \\
\hline Place of residence & & & & & $p>0.05$ (NS) \\
\hline Urban & 35 & 77.7 & 33 & 73.3 & \\
\hline Rural & 10 & 22.2 & 12 & 26.66 & \\
\hline
\end{tabular}

Abbreviation: NS, not significant. 
Table 2 Risk factors related to the COPD patients and their comparison between the two groups

\begin{tabular}{|c|c|c|c|c|c|}
\hline \multirow[t]{2}{*}{ Variables } & \multicolumn{2}{|c|}{ Supplement group $(n=45)$} & \multicolumn{2}{|c|}{ Placebo group $(n=45)$} & \multirow[t]{2}{*}{$p$-value } \\
\hline & Number & Percentage & Number & Percentage & \\
\hline \multicolumn{3}{|c|}{ Having history of smoking cessation } & & & $>0.05$ (NS) \\
\hline Positive & 12 & 26.7 & 20 & 44.4 & \\
\hline Negative & 33 & 73.3 & 25 & 55.6 & \\
\hline \multicolumn{3}{|c|}{ Having history of baking bread } & & & $>0.05$ (NS) \\
\hline Positive & 2 & 4.44 & I & 2.22 & \\
\hline Negative & 43 & 95.5 & 44 & 97.7 & \\
\hline \multicolumn{3}{|c|}{ Having history of exacerbation } & & & $>0.05$ (NS) \\
\hline Positive & 25 & 55.5 & 24 & 53.3 & \\
\hline Negative & 20 & 44.4 & 21 & 46.6 & \\
\hline
\end{tabular}

Abbreviation: NS, not significant.

education level, occupational levels, smoking history and abandonment, and the number of exacerbations) between the two groups. The mean age of supplement and placebo groups was $63.60 \pm 10.94$ (mean $\pm \mathrm{SD}$ ) years and $61.64 \pm 10.60$ years, respectively. The results indicated that there was no significant difference between the two groups regarding serum level of MDA at the beginning and end of the study. However, paired sample $t$-test revealed that the serum level of MDA decreased significantly at the end of the 6th week compared to that at the beginning of the study ( $p=0.0004)$ in the supplement group, while in the placebo group, the difference was found to be insignificant ( $p=0.3$; Table 3 ). There were no significant differences in the serum levels of MMP2 between the two groups at the beginning and end of the study. The serum level of MMP2 decreased and increased insignificantly in supplement and placebo groups, respectively (Table 4). The results of the analysis of MMP9 levels revealed that at the beginning of the study, the two groups were not significantly different in this regard. However, the difference turned out to be significant at the end of the study $(p<0.05)$. Paired sample $t$-test indicated that at the end of the 6th week, the serum level of MMP9 decreased significantly in the supplement group, while in the placebo group, its level increased significantly as compared to the beginning of the study ( $p<0.05$; Table 4$)$.

Table 3 The average level of MDA in the supplement and control groups

\begin{tabular}{lllll}
\hline Variables & $\begin{array}{l}\text { Measurement } \\
\text { stage }\end{array}$ & $\begin{array}{l}\text { Supplement } \\
\text { group } \\
(\mathbf{n}=\mathbf{4 5})\end{array}$ & $\begin{array}{l}\text { Placebo } \\
\text { group } \\
(\mathbf{n}=\mathbf{4 5})\end{array}$ & $\mathbf{p}$-value \\
\hline $\mathrm{MDA}$ & At the beginning & $0.23 \pm 0.10$ & $0.22 \pm 0.12$ & 0.07 \\
$(\mathrm{nmol} / \mathrm{mL})$ & At the end & $0.15 \pm 0.13$ & $0.27 \pm 0.17$ & 0.08 \\
$p$-value & & $0.0004^{*}$ & 0.3 & \\
\hline
\end{tabular}

Notes: $*_{p}<0.05$ in paired sample $t$-test vs baseline. The data are presented as mean \pm SD.

Abbreviation: MDA, malondialdehyde.

\section{Discussion}

The results of this study indicated that CLA significantly decreased the serum levels of MDA and MMP9 in COPD patients at the end of the 6 th week. In the placebo group, the serum levels of MMP9 increased significantly in patients. In the COPD patients, an increase in the level of oxidative stress has been observed, which might play a role in the pathogenesis of this disease. ${ }^{3}$ The increase of oxidative stress and different types of serum MMPs, especially MMP2 and MMP9, has been reported as important factors in the pathogenesis of COPD in the patients suffering from this disease. ${ }^{3}$ In COPD, body cells are constantly exposed to various oxidizing agents. Reactive oxygen species (ROS) is one of the oxidizing compounds produced in human body.,21 On the other hand, analyses in laboratory conditions have shown that the increase of oxidative stress acts as a trigger for the increase of MMPs, especially MMP2 and MMP9. ${ }^{11}$ Therefore, controlling oxidative stress and inhibiting the synthesis of MMPs are important in the treatment of this disease and can be considered as a new therapeutic strategy. ${ }^{22}$ Although there are few studies about the effect of CLA on different cancers and some chronic diseases, ${ }^{15,23}$ very limited

Table 4 The average serum levels of MMP2 and MMP9 in the supplement and placebo groups

\begin{tabular}{lllll}
\hline Variables & $\begin{array}{l}\text { Measurement } \\
\text { stage }\end{array}$ & $\begin{array}{l}\text { Supplement } \\
\text { group } \\
(\mathbf{n}=\mathbf{4 5})\end{array}$ & $\begin{array}{l}\text { Placebo } \\
\text { group } \\
(\mathbf{n}=\mathbf{4 5})\end{array}$ & -value \\
\hline $\mathrm{MMP2}$ & At the beginning & $986.27 \pm 108$ & $996.27 \pm 75$ & 0.6 \\
$(\mathrm{ng} / \mathrm{mL})$ & At the end & $980.33 \pm 100$ & $1005 \pm 77$ & 0.19 \\
$p$-value & & 0.11 & 0.16 & \\
MMP9 & At the beginning & $827 \pm 113$ & $849 \pm 149$ & 0.4 \\
$(\mathrm{ng} / \mathrm{mL})$ & At the end & $735 \pm 104$ & $875 \pm 135$ & $0.00^{\mathrm{a}}$ \\
$p$-value & & $0.00 \mathrm{I}^{\mathrm{b}}$ & $0.00 \mathrm{I}^{\mathrm{b}}$ & \\
\hline
\end{tabular}

Notes: ${ }^{a} p<0.05$ in independent samples $t$-test between two groups; ${ }^{b} p<0.05$ in paired sample $t$-test vs baseline. The data are presented as mean \pm SD. Abbreviation: MMP, matrix metalloproteinase. 
investigations have been conducted about the effect of this supplement on COPD patients.

The results of this study are consistent with the findings of the studies conducted by Baghi et al on healthy males, ${ }^{16}$ Hassan Eftekhari et al on cardiovascular patients, ${ }^{24}$ and Aryaeian et al on patients with rheumatoid arthritis. ${ }^{25}$ In their studies, they indicated that the consumption of CLA supplement can decrease the serum level of MDA, which is an index of oxidative stress. The reason for the decrease in the serum level of MDA might be the anti-inflammatory effect of CLA as the result of the activation of peroxisome proliferator-activated receptors. Through this process, CLA finally leads to the inhibition of some key factors involved in the induction of oxidative stress and triggers the production of some proinflammatory factors. ${ }^{26-28}$ During the present study, the serum level of MDA significantly decreased in the supplement group, while in the placebo group, its level slightly increased. Antioxidant enzymes and compounds are produced in the body to cope with oxidative stress conditions ${ }^{21}$ so as to protect the cells and living tissues of the body against the damages caused by free radicals and ROS. ${ }^{3}$ Therefore, treatment with nutritional antioxidants might be helpful. In other study, Shadman et al found that the consumption of CLA did not change the serum level of MDA significantly in patients with type II diabetes. ${ }^{29}$ Their finding is inconsistent with the results of our study, the possible reasons for which might be the different statistical populations addressed in each study and the increase of resistance to insulin and peroxidation lipids as the result of CLA consumption in patients with diabetes. ${ }^{29}$

In the present study, the serum concentration of MMP9 significantly decreased in COPD patients after the consumption of CLA, which shows the positive effect of this supplement. Our results are in line with the findings of the studies conducted by Hubbard et al, ${ }^{30}$ Harris et al, ${ }^{31}$ and Soel et al, ${ }^{32}$ who found that the consumption of CLA can decrease the serum level of MMP9 in healthy and cancerous rats. Although the exact mechanism of action of CLA on MMP2 and MMP9 in COPD patients is unknown, some researchers have reported that it can affect the gene expression of MMP2 and MMP9 and suppress their activity via stopping the production of mRNAs. ${ }^{30}$ An evidence for this explanation can come from the study of Hubbard et al in which they observed the destruction of mRNAs of these MMPs in rats. ${ }^{30}$ Another mechanism may be related to the regulation of gene expression of MMPs by cytokines. ${ }^{22}$ Probably, the consumption of CLA decreases the production of MMPs via the inhibition of cytokines. ${ }^{33}$ Finally, the third mechanism of action is the decrease in the production of oxidative stress considering the fact that MMPs alone are inactive and it is the oxidative stress that activates them. ${ }^{6}$ Platt et al have shown $^{34}$ that the CLA did not affect the level of MMP9, which is inconsistent with our findings. This inconsistency might be due to the different settings in which the study has been conducted and also the individual differences of the patients under study, such as age, gender, and having other diseases, and the duration of the intervention should also be considered. ${ }^{11}$

The duration of intervention and assessment of CLA blood levels in the patients were the main limitations of this study.

\section{Conclusion}

The serum levels of MDA and MMP9 decreased significantly, and an insignificant decrease in the serum level of MMP2 at the end of the 6th week after CLA supplementation was observed. These findings indicated that CLA supplementation may be helpful for COPD patients through inhibiting the production of oxidative stress and controlling MMP9 serum levels.

\section{Acknowledgments}

We are grateful to Ardabil University of Medical Sciences for financial support, to all the subjects for their participation, and to the Vice-Chancellor's Office for allowing us to conduct this study at Ardabil University of Medical Sciences and for help in data collection.

\section{Disclosure}

The authors report no conflicts of interest in this work.

\section{References}

1. Pauwels RA, Rabe KF. Burden and clinical features of chronic obstructive pulmonary disease (COPD). Lancet. 2004;364(9434):613-620.

2. Rahman I. The role of oxidative stress in the pathogenesis of COPD: implications for therapy. Treat Rspir Med. 2005;4(3):175-200.

3. Lipinski B. Hydroxyl radical and its scavengers in health and disease. Oxid Med Cell Longev. 2011;2011:809696.

4. Bowler RP, Barnes PJ, Crapo JD. The role of oxidative stress in chronic obstructive pulmonary disease. COPD. 2004;1(2):255-277.

5. Kirkham P, Rahman I. Oxidative stress in asthma and COPD: antioxidants as a therapeutic strategy. Pharmacol Ther. 2006;111(2):476-494.

6. Churg A, Zhou S, Wright JL. Series "matrix metalloproteinases in lung health and disease": matrix metalloproteinases in COPD. Eur Respir J. 2012;39(1):197-209.

7. Mercer PF, Shute JK, Bhowmik A, Donaldson GC, Wedzicha JA, Warner JA. MMP-9, TIMP-1 and inflammatory cells in sputum from COPD patients during exacerbation. Respir Res. 2005;6(1):151-155.

8. Navratilova Z, Kolek V, Petrek M. Matrix metalloproteinases and their inhibitors in chronic obstructive pulmonary disease. Arch Immunol Therap Exp. 2016;64(3):177-193. 
9. Brajer B, Batura-Gabryel H, Nowicka A, Kuznar-Kaminska B, Szczepanik A. Concentration of matrix metalloproteinase-9 in serum of patients with chronic obstructive pulmonary disease and a degree of airway obstruction and disease progression. J Physiol Pharmacol. 2008; 59(Suppl 6):145-152.

10. Agha-Hosseini F, Mirzaii-Dizgah I, Farmanbar N, Abdollahi M. Oxidative stress status and DNA damage in saliva of human subjects with oral lichen planus and oral squamous cell carcinoma. J Oral Pathol Med. 2012;41(10):736-740.

11. Belvisi MG, Bottomley KM. The role of matrix metalloproteinases (MMPs) in the pathophysiology of chronic obstructive pulmonary disease (COPD): a therapeutic role for inhibitors of MMPs? Inflamm Res. 2003;52(3):95-100.

12. Bhattacharya A, Banu J, Rahman M, Causey J, Fernandes G. Biological effects of conjugated linoleic acids in health and disease. $J$ Nutr Biochem. 2006;17(12):789-810.

13. Ing SW, Belury MA. Impact of conjugated linoleic acid on bone physiology: proposed mechanism involving inhibition of adipogenesis. Nutr Rev. 2011;69(3):123-131.

14. Raff M, Tholstrup T, Basu S, Nonboe P, Sorensen MT, Straarup EM. A diet rich in conjugated linoleic acid and butter increases lipid peroxidation but does not affect atherosclerotic, inflammatory, or diabetic risk markers in healthy young men. $J$ Nutr. 2008;138(3):509-514.

15. Tholstrup T, Raff M, Straarup EM, Lund P, Basu S, Bruun JM. An oil mixture with trans-10, cis-12 conjugated linoleic acid increases markers of inflammation and in vivo lipid peroxidation compared with cis-9, trans-11 conjugated linoleic acid in postmenopausal women. J Nutr. 2008; 138(8):1445-1451.

16. Baghi AN, Mazani M, Nemati A, Amani M, Alamolhoda S, Mogadam RA. Anti-inflammatory effects of conjugated linoleic acid on young athletic males. JPMA J Pak Med Assoc. 2016;66(3):280-284.

17. Oraldi M, Maggiora M, Paiuzzi E, Canuto RA, Muzio G. CLA reduces inflammatory mediators from A427 human lung cancer cells and A427 conditioned medium promotes differentiation of $\mathrm{C} 2 \mathrm{C} 12$ murine muscle cells. Lipids. 2013;48(1):29-38.

18. Khoubnasabjafari M, Ansarin K, Jouyban A. Reliability of malondialdehyde as a biomarker of oxidative stress in psychological disorders. BioImpacts. 2015;5(3):123.

19. Bajpai J, Prakash V, Kant S, et al. Study of oxidative stress biomarkers in chronic obstructive pulmonary disease and their correlation with disease severity in north Indian population cohort. Lung India. 2017; 34(4):324.

20. Zeng M, Li Y, Jiang Y, Lu G, Huang X, Guan K. Local and systemic oxidative stress status in chronic obstructive pulmonary disease patients. Can Respir J. 2013;20(1):35-41.

21. Mittler R. Oxidative stress, antioxidants and stress tolerance. Trends in Plant Science. 2002;7(9):405-410.
22. Roomi MW, Monterrey JC, Kalinovsky T, Rath M, Niedzwiecki A. Patterns of MMP-2 and MMP-9 expression in human cancer cell lines. Oncol Rep. 2009;21(5):1323-1333.

23. Yang M, Cook ME. Dietary conjugated linoleic acid decreased cachexia, macrophage tumor necrosis factor-alpha production, and modifies splenocyte cytokines production. Exp Biol Med. 2003;228(1):51-58.

24. Hassan Eftekhari M, Aliasghari F, Babaei-Beigi MA, Hasanzadeh J. Effect of conjugated linoleic acid and omega-3 fatty acid supplementation on inflammatory and oxidative stress markers in atherosclerotic patients. ARYA Atheroscler. 2013;9(6):311-318.

25. Aryaeian N, Djalali M, Shahram F, Djazayery A, Eshragian MR. Effect of conjugated linoleic acid, vitamin E, alone or combined on immunity and inflammatory parameters in adults with active rheumatoid arthritis: a randomized controlled trial. Int J Prev Med. 2014;5(12): $1567-1577$

26. Butz DE, Li G, Huebner SM, Cook ME. A mechanistic approach to understanding conjugated linoleic acid's role in inflammation using murine models of rheumatoid arthritis. Am J Physiol Regul Integr Comp Physiol. 2007;293(2):R669-R676.

27. Mohammadzadeh M, Faramarzi E, Mahdavi R, Nasirimotlagh B, Asghari Jafarabadi M. Effect of conjugated linoleic acid supplementation on inflammatory factors and matrix metalloproteinase enzymes in rectal cancer patients undergoing chemoradiotherapy. Integr Cancer Ther. 2013;12(6):496-502.

28. Park NY, Valacchi G, Lim Y. Effect of dietary conjugated linoleic acid supplementation on early inflammatory responses during cutaneous wound healing. Mediators Inflamm. 2010;2010. Pii: 342328

29. Shadman Z, Taleban FA, Saadat N, Hedayati M. Effect of conjugated linoleic acid and vitamin $\mathrm{E}$ on glycemic control, body composition, and inflammatory markers in overweight type 2 diabetics. J Diabetes Metab Disord. 2013;12(1):42.

30. Hubbard NE, Lim D, Erickson KL. Conjugated linoleic acid alters matrix metalloproteinases of metastatic mouse mammary tumor cells. J Nutr. 2007;137(6):1423-1429.

31. Harris MA, Hansen RA, Vidsudhiphan P, et al. Effects of conjugated linoleic acids and docosahexaenoic acid on rat liver and reproductive tissue fatty acids, prostaglandins and matrix metalloproteinase production. Prostaglandins Leukot Essent Fatty Acids. 2001;65(1):23-29.

32. Soel SM, Choi OS, Bang MH, Yoon Park JH, Kim WK. Influence of conjugated linoleic acid isomers on the metastasis of colon cancer cells in vitro and in vivo. $J$ Nutr Biochem. 2007;18(10):650-657.

33. Ghobadi H, Matin S, Nemati A, Naghizadeh-Baghi A. The effect of conjugated linoleic acid supplementation on the nutritional status of COPD patients. Int J Chron Obstruct Pulmon Dis. 2016;11:2711-2720.

34. Platt I, El-Sohemy A. Effects of 9 cis, 11 trans and 10 trans, 12 cis CLA on osteoclast formation and activity from human CD14+ monocytes. Lipids Health Dis. 2009;8(1):15.
International Journal of COPD

\section{Publish your work in this journal}

The International Journal of COPD is an international, peer-reviewed journal of therapeutics and pharmacology focusing on concise rapid reporting of clinical studies and reviews in COPD. Special focus is given to the pathophysiological processes underlying the disease, intervention programs, patient focused education, and self management protocols.

\section{Dovepress}

This journal is indexed on PubMed Central, MedLine and CAS. The manuscript management system is completely online and includes a very quick and fair peer-review system, which is all easy to use. Visit $\mathrm{http}: / / \mathrm{www}$. dovepress.com/testimonials.php to read real quotes from published authors. 